\title{
Human Pluripotent Stem Cell-Derived Hepatocytes Show Higher Transcriptional Correlation with Adult Liver Tissue than with Fetal Liver Tissue
}

Nidal Ghosheh,* Barbara Küppers-Munther, Annika Asplund, Christian X. Andersson, Petter Björquist, Tommy B. Andersson, Helena Carén, Stina Simonsson, Peter Sartipy, and Jane Synnergren

Cite This: ACS Omega 2020, 5, 4816-4827

Read Online

ACCESS | Lلll Metrics \& More | 国 Article Recommendations | s supporting Information

ABSTRACT: Human pluripotent stem cell-derived hepatocytes (hPSC-HEP) display many properties of mature hepatocytes, including expression of important genes of the drug metabolizing machinery, glycogen storage, and production of multiple serum proteins. To this date, hPSC-HEP do not, however, fully recapitulate the complete functionality of in vivo mature hepatocytes. In this study, we applied versatile bioinformatic algorithms, including functional annotation and pathway enrichment analyses, transcription factor binding-site enrichment, and similarity and correlation analyses, to datasets collected from different stages during hPSC-HEP differentiation and compared these to developmental stages and tissues from fetal and adult human liver. Our results demonstrate a high level of similarity between the in vitro differentiation of hPSC-HEP and in vivo hepatogenesis. Importantly, the transcriptional correlation of hPSC-HEP with adult liver (AL) tissues was higher than with fetal liver (FL) tissues ( 0.83 and 0.70 , respectively). Functional data revealed mature features of hPSC-HEP including cytochrome P450 enzymes activities and albumin

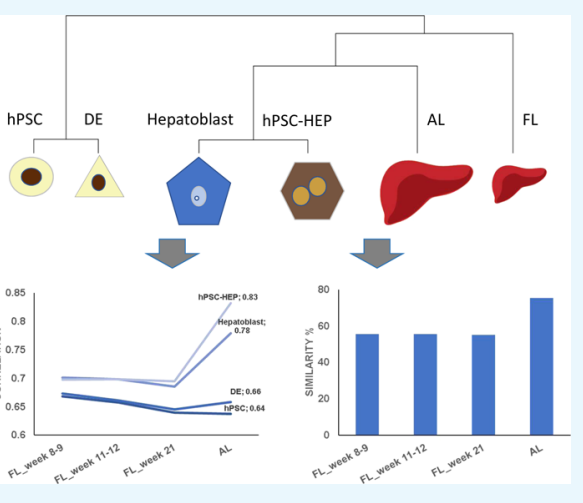
secretion. Moreover, hPSC-HEP showed expression of many genes involved in drug absorption, distribution, metabolism, and excretion. Despite the high similarities observed, we identified differences of specific pathways and regulatory players by analyzing the gene expression between hPSC-HEP and AL. These findings will aid future intervention and improvement of in vitro hepatocyte differentiation protocol in order to generate hepatocytes displaying the complete functionality of mature hepatocytes. Finally, on the transcriptional level, our results show stronger correlation and higher similarity of hPSC-HEP to AL than to FL. In addition, potential targets for further functional improvement of hPSC-HEP were also identified.

\section{INTRODUCTION}

Human pluripotent stem cell-derived hepatocytes (hPSC$\mathrm{HEP}$ ) are an attractive cell source for in vitro applications, such as disease modeling, studies on drug metabolism and drug targets, safety assessment, viral studies, and also for use in regenerative medicine. Currently used primary hepatocytes suffer from limited availability, short life span, dedifferentiation, and loss of important metabolic functions in vitro. hPSC-HEP share many features with their in vivo counterparts, and recent improvement of differentiation protocols simulating liver development has allowed the generation of cells with improved hepatocyte functionality. ${ }^{1}$ However, to completely replace the currently used cell sources, the hPSC-HEP need further refinement. $^{2,3}$

There are reports in the literature describing knowledgebased approaches for functional improvement of hPSC-HEP. For instance, Zhao et al. observed that hepatoblasts obtained according to a previous hepatocyte differentiation protocol did not express hepatocyte nuclear protein 6 (HNF6) and prospero homeobox 1 (PROX1), as would be expected during liver development. They then modified their differentiation protocol, which resulted in the expression of HNF6 and PROX1 in addition to HNF1A and CCAAT enhancer binding protein alpha (CEBPA) and the subsequently improved expression of transporters and drug-metabolizing enzymes in the produced hepatocytes. ${ }^{1}$ Other researchers reported that hPSC-HEP have mixed features of liver, intestine, fibroblast, and stem cells, ${ }^{2}$ or that hPSC-HEP have major similarity to fetal liver (FL), rather than to adult liver $(\mathrm{AL}){ }^{4}$

Most of the liver volume $(70-85 \%)$ is made up by hepatocytes, ${ }^{5}$ and these cells are responsible for key liver functions, including protein secretion, bile secretion, detoxification, urea metabolism, glucose/glycogen metabolism and storage, and cholesterol metabolism as well as secretion of

Received: October 21, 2019

Accepted: February 14, 2020

Published: March 2, 2020 
clotting factors and acute phase response. ${ }^{6}$ Detoxification is a major function of hepatocytes and is conducted by a set of proteins involved in absorption, distribution, metabolism, and excretion (ADME) of compounds, including phase I and phase II xenobiotic metabolizing enzymes and phase III transporters. The ADME functionality is of high importance when considering the employment of hPSC-HEP in drug discovery and ToxSafety. However, several ADME genes have, until now, shown low or no expression in hPSC-HEP. Therefore, improving the expression of these genes in hPSC-HEP is of critical importance for such applications.

In the present study, we analyzed transcriptomics data from one dataset, including hPSC-HEP generated by a standardized protocol, $^{7-9}$ with human $\mathrm{AL}$ tissue as the control, and integrated this with the data obtained from human FL and $\mathrm{AL}$ tissues from another dataset to identify similarities and differences between in vivo- and in vitro-derived hepatocytes. Importantly, the hPSC-HEP analyzed in this study showed higher transcriptional similarity and correlation with AL than with FL. Moreover, the functionality of hPSC-HEP was confirmed by measuring the drug-metabolizing activity of cytochrome P450 (CYP) enzymes, the secretion of albumin, and the production of urea. In addition, many pathways involved in the functionality of hepatocytes and important transcription factors (TFs) regulating hepatocyte maturity were identified in the hPSC-HEP by applying bioinformatic analysis on our generated transcriptomics dataset including both hPSC-HEP and two biological control samples from AL.

\section{RESULTS}

Mapping of in Vitro Differentiation to in Vivo Developmental Stages. In order to map the different stages of in vitro differentiation to different stages of in vivo liver development, data from FL and AL from the Gene Expression Omnibus (GEO) database (accession number: GSE61279) were merged with our dataset (E-MTAB-5367), and the hPSCHEP were mapped to the corresponding in vivo developmental stages based on correlation with the gene expression. Only FL from male human embryonic development at gestation weeks $8-9,11-12$, and 21 and AL from males of age $22-38$ years (with accident as cause of death) were selected from the GSE61279 dataset and merged with our transcriptional dataset from in vitro hepatocyte differentiation. In total, 12922 genes were included in the merged dataset. To reduce batch effects between these datasets, the merged dataset was normalized by applying the COMBAT software. ${ }^{10}$ Boxplots and hierarchical clustering analysis of the merged and normalized datasets validated successful integration of the two datasets (Figures S1 and 1 respectively). Interestingly, the results showed clustering of hepatoblasts and hPSC-HEP on day 25 closer to AL than to FL (Figure 1).

Both the correlation analysis and the analysis of similarity on the gene expression level were performed with concordant results. First, Spearman's correlation was calculated between each in vitro time point and the FL at weeks 8-9, 11-12, and 21 as well as the AL. Remarkably, the results showed that in vitro-derived hPSC-HEP $\mathrm{d} 25$ and hepatoblasts have higher correlation with AL ( 0.83 and 0.78 , respectively) than with FL $(\leq 0.70) \quad(P$-value $<0.001)$ (Figure $2 \mathrm{~A})$. Furthermore, the correlation results between AL and FL at weeks $8-9,11-12$, and 21 were $0.69,0.70$, and 0.72 , respectively ( $P$-value < $0.001)$, demonstrating that the transcriptional differences

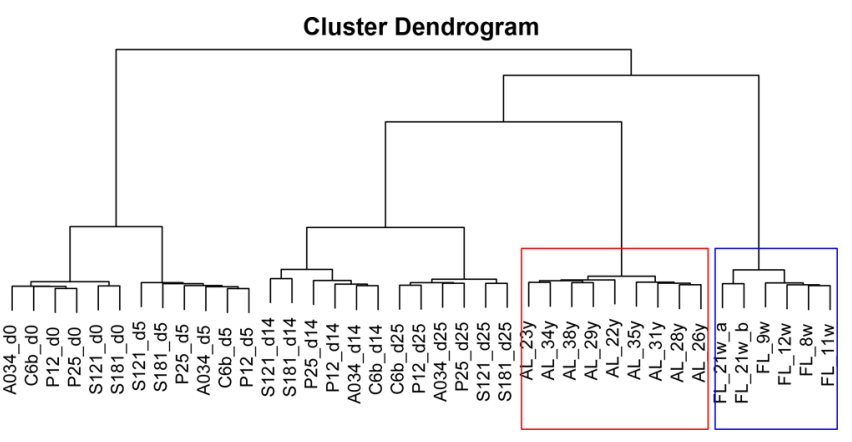

Figure 1. Hierarchical clustering of the merged dataset grouped data into distinct clusters of hPSC (d0), DE (d5), hepatoblasts (d14), hPSC-HEP (d25), human FL tissues, and human AL tissues. AL_26y and AL_29y in the red marked cluster of AL samples are from the EMTAB-5367 dataset, and the rest of the AL samples are from the GSE61279 dataset. Despite different platforms used, all nine samples from the adult liver clustered tight together.

between these two control groups are distinct and reflected in the correlation analysis.

Next, a similarity analysis based on gene expression levels, as described in the methods, was performed between hPSC-HEP and the FL and AL. In concordance with results from the correlation analysis, these results showed that hPSC-HEP d25 have greater similarity with $\mathrm{AL}(75 \%)$ than with FL $(\leq 56 \%)$ (Figure 2B). Notably, the similarity of AL to the different FL tissues was lower or equal to $63 \%$.

Identification of Differentially Expressed Genes during Hepatocyte Differentiation and between hPSCHEP and AL. To identify differentially expressed genes between each developmental stage during the stepwise hepatocyte differentiation and in comparison to AL, the significance analysis of microarray (SAM) data algorithm was applied to the transitions hPSC-DE, DE-hepatoblasts, hepatoblasts-hPSC-HEP day 25, hPSC-HEP day 25-hPSCHEP day 30, and hPSC-HEP day 25-AL in the dataset EMTAB-5567 (Figure 9). A combined threshold of false discovery rate $(\mathrm{FDR})<0.05$ and fold change $(\mathrm{FC})>3$ was used to identify the significant differential expression. The largest number (1032) of differentially expressed genes during the hepatocyte differentiation was observed between DE and hepatoblasts, of which 223 were downregulated in hepatoblasts. Notably, only 11 genes were differentially expressed between hPSC-HEP day 25 and hPSC-HEP day 30. Further, 1078 genes were differentially expressed between hPSC-HEP day 25 and $\mathrm{AL}$, of which 582 genes were downregulated in $\mathrm{AL}$ (Table S1). As a first step to analyze the differentially expressed genes, genes with exceptionally high FC levels (FC $\geq 20$ ) were identified and further explored. The highest FC (272) in the comparison hPSC versus DE was shown for the DE marker Cerberus 1 (CER1). C-X-C motif chemokine receptor 4 (CXCR4), another important DE marker, was also strongly upregulated. Interestingly, an unknown transcript, RP4-559A3.6, with FC of 175 was highly upregulated in the DE cells compared to hPSC. Among the highly upregulated genes in hPSCs compared to DE were the noncoding transcript JAKMIP2-AS1 in addition to the genes apelin receptor early endogenous ligand (APELA), protein tyrosine phosphatase receptor Z1 (PTPRZ1), and SAM domain and HD domain 1 (SAMHD1). The top five coding transcripts with the highest FC values that showed upregulation in hepatoblasts compared to DE cells were the fibrinogen alpha 

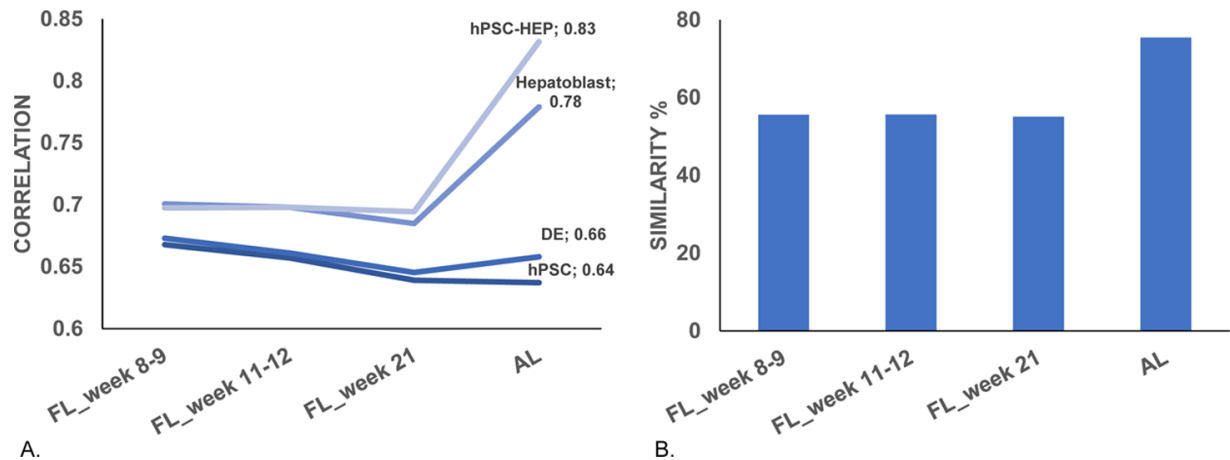

Figure 2. (A) Spearman's correlation results for hPSC, DE, hepatoblasts, and hPSC-HEP day 25 with FL week 8-9, FL week 11-12, FL week 21, and AL (confidence interval 95\%). (B) Similarity analysis results for hPSC-HEP day 25 to FL week 8-9, FL week 11-12, FL week 21, and AL for $\mathrm{FC}<1.5$ between mean values of mentioned comparisons and coefficient of variance $<30 \%$ across replicated samples.

chain $(F G A)$, apolipoprotein $\mathrm{B}(A P O B)$, alpha fetoprotein $(A F P)$, fibrinogen beta chain $(F G B)$, and fibrinogen gamma chain (FGG) with FC values of $265,208,185,166$, and 143, respectively. Mucin 13 (MUC13) was upregulated in hPSCHEP day 25 compared to hepatoblasts with an FC value of 133, in addition to leucine-rich repeat-containing protein 19 (LRRC19), lactase (LCT), annexin A13 (ANXA13), and cadherin $17(\mathrm{CDH} 17)$ that also showed $\mathrm{FC}$ values $>55$. However, these genes were downregulated in $\mathrm{AL}$. In the comparison of hPSC-HEP day 25 versus hPSC-HEP day 30, no genes with FC > 20 were observed (Table S2).

The genes, C-reactive protein $(C R P)$, coagulation factor IX (F9), complement factor $\mathrm{H}$ related 2 (CFHR2), complement C9 (C9), and alcohol dehydrogenase 1B ( $A D H 1 B)$, were upregulated in $\mathrm{AL}$, compared to hPSC-HEP day 25, with extraordinary high FC values ranging from 132 to 280 . On the other hand, the genes LCT, AFP, CDH17, MUC13, and hepatitis A virus cellular receptor 1 (HAVCR1) were among the highly downregulated genes in AL compared to hPSC-HEP day 25 (Table S2).

Because the liver plays an important role in the metabolism of xenobiotics, the expression of ADME genes in hepatocytes is of great interest. Noteworthy, we found that $22.4 \%$ of the total ADME genes were downregulated in hPSC-HEP compared to AL. Sixteen of the 32 core ADME genes (http://www. pharmaadme.org) were differentially expressed between hPSCHEP day 25 and AL, whereas solely cytochrome P450 1A1 (CYP1A1) and ABCG2, a member of the ATP-binding cassette (ABC) transporters, were upregulated in hPSC-HEP. In addition, the genes, solute carrier organic anion transporter family member 1B1 (SLCO1B1), SLCO1B3, CYP2A6, CYP2B6, CYP2C8, CYP2C9, and CYP2E1, were among the most upregulated genes in AL compared to hPSC-HEP day 25. In the extended $\mathrm{ADME}$ gene list, 73 of $267 \mathrm{ADME}$ genes were differentially expressed, of which 20 genes were upregulated in hPSC-HEP day 25 including CYP1B1, CYP2S1, CYP24A1, SLC10A2, SLC15A1, UDP glycosyltransferase 8 (UGT8), glutathione $S$-transferase alpha 1 (GSTA1), GSTA2, GSTA3, and flavin containing monooxygenase (FMO1). The genes $A D H 1 A, A D H 1 B, A D H 1 C$, carboxylesterase 1 (CES1), aldehyde oxidase 1 (AOX1), and paraoxonase 1 (PON1) showed the highest upregulation in AL compared to hPSCHEP day 25. Surprisingly, the fetal enzyme CYP $3 A 7$ showed higher expression level in AL than in hPSC-HEP day 25 (Table S3).
Only 10 core ADME genes were differentially expressed between hepatoblasts and hPSC-HEP day 25. These genes, including CYP3A4, CYP2C9, and CYP2B6, were upregulated in hPSC-HEP day 25. However, CYP2C9 and CYP2B6 were still low-expressed in hPSC-HEP day 25 compared to AL, whereas CYP3A4 did not show significant differential expression between AL and hPSC-HEP. The genes CYP1Al and $A B C G 2$ were downregulated in hepatoblasts and $\mathrm{AL}$ compared to hPSC-HEP day 25. In addition, 19 genes of the extended ADME list were also differentially expressed between hepatoblasts and hPSC-HEP day 25. The genes, serpin A7 (SERPINA7) and SLC22A3, were upregulated in both hepatoblasts and in AL compared to hPSC-HEP day 25 (Table S4).

Venn Diagram for the Differentially Expressed Genes. The number of overlapping differentially expressed genes at defined developmental stages during the hepatocyte differentiation is illustrated in a Venn diagram showing differentially expressed genes for the following comparisons: hPSC versus DE (d0vsd5), DE versus hepatoblasts (d5vsd14), hepatoblasts versus hPSC-HEP day 25 (d14vsd25), and hPSCHEP day 25 versus AL (d25vsAL) (Figure 3).

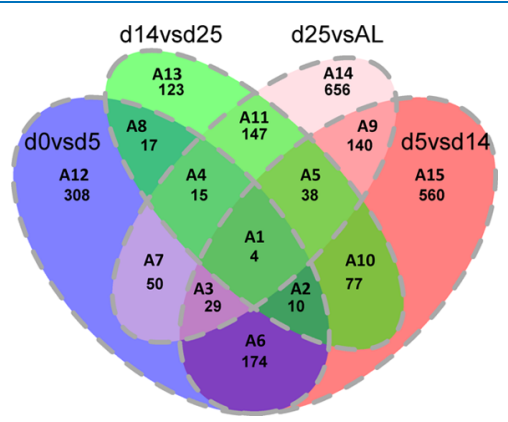

Figure 3. Venn diagram showing the number of differentially expressed genes from the following comparisons: hPSC vs DE (d0vsd5), DE vs hepatoblasts (d5vsd14), hepatoblasts vs hPSC-HEP day 25 (d14vsd25), and hPSC-HEP day 25 vs AL (d25vsAL).

Four transcripts were differentially expressed between all comparisons (region A1) including three coding transcripts, collagen type $\mathrm{V}$ alpha 2 chain (COL5A2), stanniocalcin 1 (STC1), and angiotensin I converting enzyme 2 (ACE2), and one noncoding transcript, long intergenic non-protein coding RNA 261 (LINC00261). Moreover, 10 transcripts were identified as differentially expressed in all three transitions 

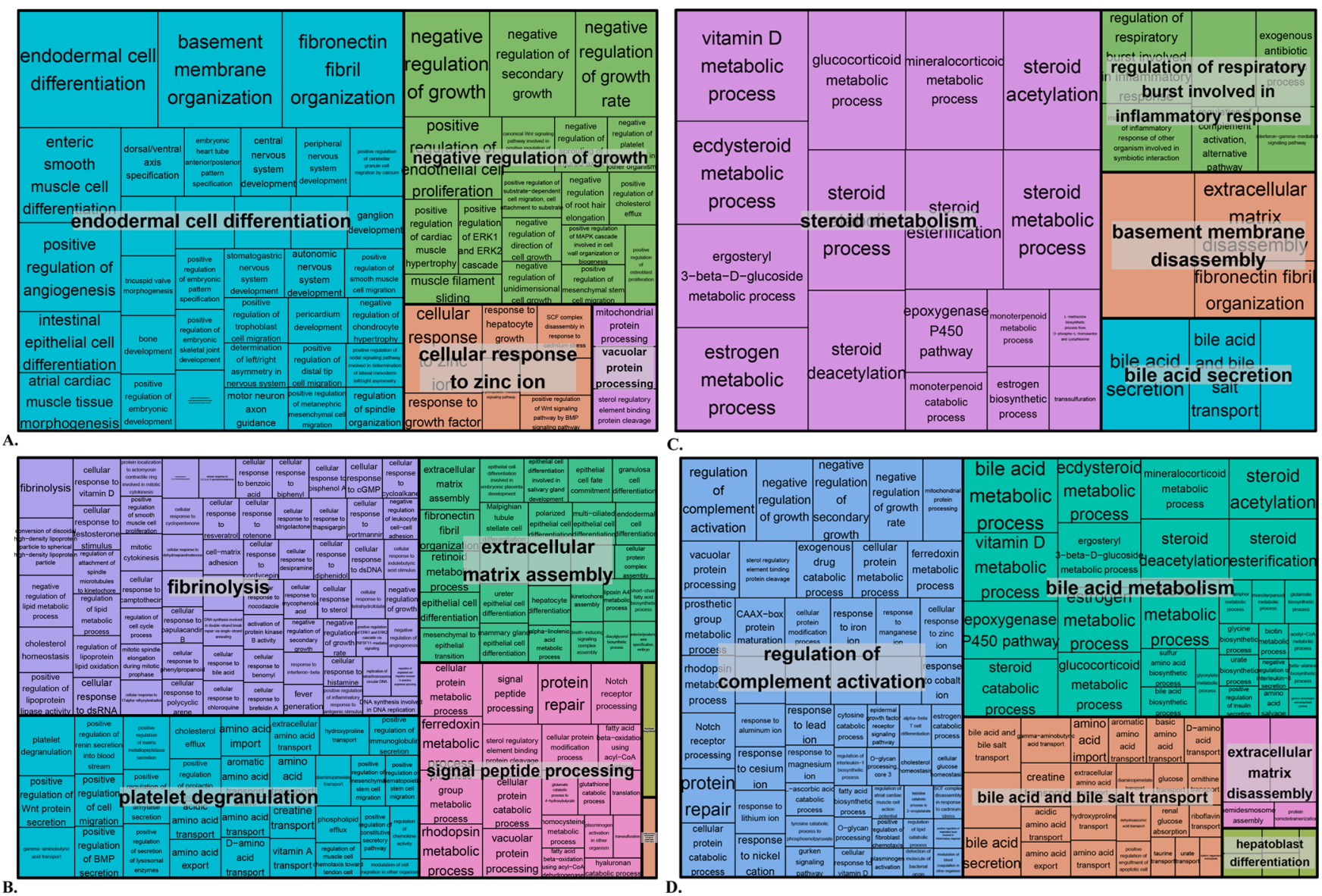

Figure 4. GO enrichment analysis for biological processes identified using the EnrichR software and visualized by the Revigo software for the following comparisons: (A) differentially expressed genes between hPSCs and DE cells, (B) differentially expressed genes between DE and hepatoblasts, (C) differentially expressed genes between hepatoblasts and hPSC-HEP day 25, and (D) differentially expressed genes between hPSC-HEP day 25 and AL.

from hPSC to hPSC-HEP day 25 (region A2). Noteworthy, APELA, dedicator of cytokinesis 11 (DOCK11), and SAMHD1 have opposite expression profiles compared to the transcripts CYP26A1 and dual specificity phosphatase 6 (DUSP6) (Figure $\mathrm{S} 2 \mathrm{~A}, \mathrm{~B})$. In addition, the transcripts Rho GTPase activating protein 28 (RHGAP28) and follistatin (FST) have opposite expression profiles compared to the transcripts stomatin (STOM) and thioredoxin interacting protein (TXNIP) (Figure S2C,D). The great majority of the genes that were differentially expressed only in the comparisons, hepatoblasts versus hPSCHEP day 25 and hPSC-HEP day 25 versus AL (region A11), were upregulated in hPSC-HEP day 25 compared to both AL and hepatoblasts (Figure S3). Out of the three TFs in the region A11, sex determining region Y-box 2 (SOX2), caudal type homeobox 2 (CDX2), and forkhead box O3 (FOXO3), only CDX2 was upregulated in hPSC-HEP day 25 compared to both $\mathrm{AL}$ and hepatoblasts with FC of 5.2 and 3.4, respectively. The differentially expressed genes representing the different regions of the Venn diagram are listed in Table S5.

GO Enrichment Analysis of the Differentially Expressed Genes. In the next step, differentially expressed genes from the comparisons, hPSC versus DE, DE versus hepatoblasts, hepatoblasts versus hPSC-HEP day 25, and hPSC-HEP day 25 versus AL, were analyzed using the EnrichR software. ${ }^{11}$ Enriched gene ontology (GO) annotation for biological process terms were retrieved, and the results were visualized using the Revigo software (Figure 4) and are detailed in Table S6. Differentially expressed genes between hPSCs versus DE cells were mostly enriched for terms of "endodermal cell differentiation" and "cellular response to zinc ions," including "positive regulation of Wnt signaling pathway by BMP signaling pathway" (Figure 4A). Biological process terms enriched for the differentially expressed genes between DE and hepatoblasts were mostly associated with "fibrinolysis" including cellular response to different stimuli, "platelet degranulation," and "extra cellular matrix assembly" (Figure 4B). Annotations including "steroid metabolism," "bile acid secretion," and "basement membrane disassembly" were significantly enriched among the differentially expressed genes between hepatoblasts and hPSC-HEP day 25 (Figure 4C). Finally, differentially expressed genes in the comparison hPSC-HEP day 25 versus AL were mostly enriched for terms of "regulation of complement activation," "bile acid metabolism," and "bile acid and bile acid transport" (Figure 4D).

Enriched Pathways among the Differentially Expressed Genes. Pathway enrichment analysis was performed on the differentially expressed genes in each comparison. Differentiation pathways for the different germ layers including "endoderm differentiation," "mesodermal commitment pathway," and "ectoderm differentiation" were significantly enriched for genes upregulated in DE compared to hPSC. Differentially expressed genes between hepatoblasts and DE cells were enriched for "cell cycle" and various differentiation pathways in addition to many hepatocyte function pathways 
such as "complement and coagulation cascades," "metapathway biotransformation," and "drug induction of bile acid pathway." Pathways enriched for the differentially expressed genes between hepatoblasts and hPSC-HEP day 25 included typical pathways for mature hepatocytes such as "oxidation by cytochrome P450," "metapathway biotransformation," "constitutive androstane receptor pathway," and "farnesoid X receptor pathway" in addition to metabolism pathways including "tamoxifen metabolism" and "folate metabolism." In the comparison hPSC-HEP day 25 versus $\mathrm{AL}$, the pathways "complement activation," "liver X receptor pathway," "aryl hydrocarbon receptor pathway," "fatty acid biosynthesis," and "phase I biotransformations, non P450" were enriched (Table S7).

TF Enrichment Analysis. To further explore important regulatory mechanisms during hepatocyte differentiation, TF binding sites enrichment analysis was performed on differentially expressed genes between the four comparisons hPSC versus DE, DE versus hepatoblasts, hepatoblasts versus hPSCHEP day 25 , and hPSC-HEP day 25 versus AL by applying the EnrichR software. ${ }^{11}$ To explore if there was an overlap across the identified enriched TF binding sites between the groups of differentially expressed genes, a Venn diagram was constructed (Figure 5). As shown in Table 1, the binding sites for the TFs,

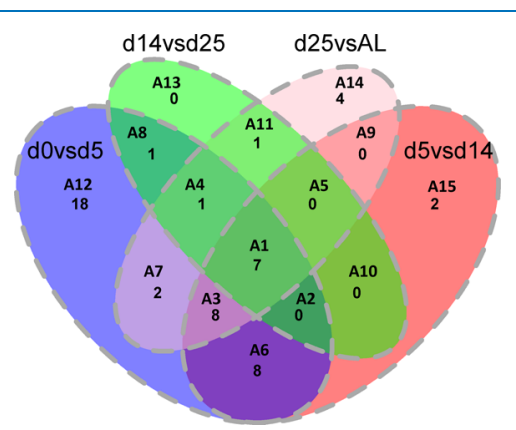

Figure 5. Venn diagram constructed for TFs with significantly enriched binding sites among the differentially expressed genes in the following transitions: hPSC-DE (d0vsd5), DE-hepatoblasts (d5vsd14), hepatoblasts-hPSC-HEP day 25 (d14vsd25), and hPSC-HEP day 25-AL (d25vsAL). See Table 1 for a list of the TFs.

Table 1. TFs Associated with the Genes in the Different Regions of the Venn Diagram (Figure 5)

\begin{tabular}{ccl} 
region & $\begin{array}{c}\text { number } \\
\text { of genes }\end{array}$ & \multicolumn{1}{c}{ genes } \\
A1 & 7 & AR; BACH1; FOXA1; FOXA2; FOXM1; JUN; SOX2 \\
A3 & 8 & $\begin{array}{l}\text { ESR2; GATA4; HNF4A; SMAD3; SMAD4; TFP53; } \\
\text { TP63; ZNF217 }\end{array}$ \\
A4 & 1 & TRIM28 \\
A6 & 8 & $\begin{array}{l}\text { EOMES; ESR1; NANOG; EP300; POU5F1; RUNX2; } \\
\text { SMAD2; UBTF }\end{array}$ \\
A7 & 2 & FOXO3; PPARA \\
A8 & 1 & ARNT \\
A11 & 1 & RELA \\
A12 & 18 & AHR; BCAT; CTBP1; CTBP2; CTNNB1; ERG; \\
& & EWSR1; GBX2; KLF5; NR3C1; PIAS1; POU3F2; \\
& & PRDM14; STAT3; TCF4; TOP2B; VDR; WT1 \\
A14 & 4 & CDX2; CLOCK; HIF1A; NR1H3 \\
A15 & 2 & E2F4; E2F7
\end{tabular}

androgen receptor (AR), BTB domain and $\mathrm{CNC}$ homolog 1 (BACH1), FOXA1, FOXA2, FOXM1, jun oncogene (JUN), and SOX2, were enriched in differentially expressed genes from all four comparisons (A1 region in the Venn diagram). The A14 region of the Venn diagram contains TFs that were differentially expressed between hPSC-HEP day 25 versus AL, and those were CDX2, clock circadian regulator (CLOCK), hypoxia inducible factor 1 subunit alpha (HIF1A), and liver X receptor alpha $(\mathrm{NR} 1 \mathrm{H} 3)$. Hence, these are of specific interest when aiming at improving hPSC-HEP.

After analyzing the enrichment of TF binding sites (Table $1)$, we investigated which of the identified TFs showed differential expression between different developmental stages in our data (Table 2). As expected, the TFs and pluripotent

Table 2. Differentially Expressed TFs Whose Binding Sites Are Enriched in the Different Developmental Stages ${ }^{a}$

\begin{tabular}{|c|c|c|c|}
\hline comparison & $\begin{array}{l}\text { gene } \\
\text { symbol }\end{array}$ & FC & description \\
\hline \multirow[t]{8}{*}{ hPSC vs DE } & EOMES & 47.4 & eomesodermin \\
\hline & FOXA2 & 3.7 & forkhead box A2 \\
\hline & GATA4 & 4.8 & GATA binding protein 4 \\
\hline & KLF5 & 4.4 & $\begin{array}{l}\text { Kruppel-like factor } 5 \\
\text { (intestinal) }\end{array}$ \\
\hline & NANOG & -3.8 & Nanog homeobox \\
\hline & POU5F1 & -7.7 & POU class 5 homeobox 1 \\
\hline & PRDM14 & -11.2 & PR domain containing 14 \\
\hline & SOX2 & -17.6 & $\begin{array}{l}\text { SRY (sex determining } \\
\text { region Y)-box } 2\end{array}$ \\
\hline \multirow[t]{4}{*}{ DE vs hepatoblasts } & AHR & 6.1 & aryl hydrocarbon receptor \\
\hline & E2F7 & -3.1 & E2F TF 7 \\
\hline & EOMES & -47.0 & eomesodermin \\
\hline & NANOG & -7.7 & Nanog homeobox \\
\hline \multirow{2}{*}{$\begin{array}{l}\text { hepatoblasts vs } \\
\text { hPSC-HEP day } 25\end{array}$} & $\mathrm{CDX} 2$ & 3.4 & caudal type homeobox 2 \\
\hline & KLF5 & 3.2 & $\begin{array}{l}\text { Kruppel-like factor } 5 \\
\text { (intestinal) }\end{array}$ \\
\hline \multirow{3}{*}{$\begin{array}{l}\text { hPSC-HEP day } 25 \text { vs } \\
\text { AL }\end{array}$} & AR & 4.4 & $\mathrm{AR}$ \\
\hline & $\mathrm{CDX} 2$ & -5.2 & caudal type homeobox 2 \\
\hline & KLF5 & -5.9 & $\begin{array}{l}\text { Kruppel-like factor } 5 \\
\text { (intestinal) }\end{array}$ \\
\hline
\end{tabular}

${ }^{a} \mathrm{FC}>0$ upregulation in the second developmental stage in the comparison; FC $<0$ upregulation in the first developmental stage in the comparison.

markers Nanog homeobox (NANOG) and POU class 5 homeobox 1 (POU5F1) were downregulated in the DE cells compared to the hPSCs, whereas NANOG was further decreasing in the transition of hepatoblasts. Another important stem cell marker, TF SOX2, is, as expected, also strongly downregulated in the comparison hPSC versus DE. Interestingly, the mesendodermal marker eomesodermin (EOMES) is first strongly upregulated in the comparison hPSC versus DE and then downregulated in the subsequent step (DE vs hepatoblasts). Two known endodermal markers, FOXA2 and GATA binding protein 4 (GATA4), were also found to be upregulated in DE compared to hPSCs. Moreover, Kruppellike factor 5 (KLF5) was upregulated in $\mathrm{DE}$, compared to hPSCs, and in hPSC-HEP day 25 compared to both hepatoblasts and AL. CDX2 was also upregulated in hPSCHEP day 25 compared to both hepatoblasts and AL. In addition, the TF $A R$ was differentially expressed in the comparison hPSC-HEP day 25 versus AL as well (Table 2).

Assessment of the Functionality of hPSC-HEP. Because we found a high correlation and similarity between hPSC-HEP and AL, we performed several functional assays 


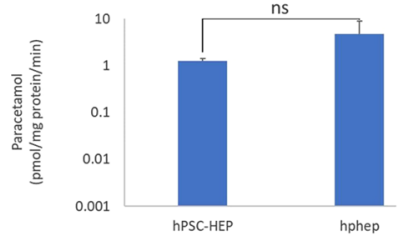

A.

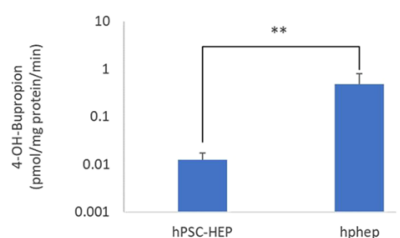

D.

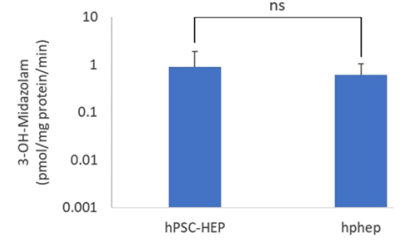

B.

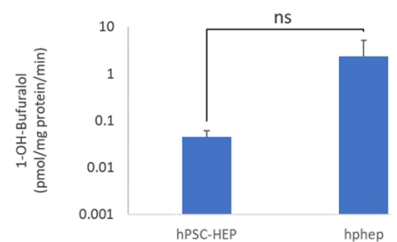

E.

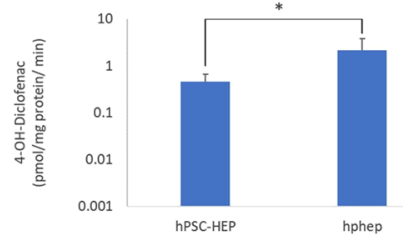

c.

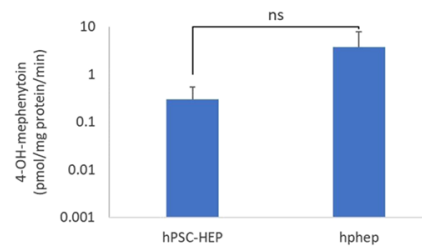

F.

Figure 6. Cytochrome P450 (CYP) activities in hPSC-HEP ( $N=6$ cell lines) on day 29 of the differentiation and cryopreserved human primary hepatocyte (hphep) cultured for $20 \mathrm{~h}$ post-thaw/plating $(\mathrm{N}=4$ donors). The concentrations of the metabolites, paracetamol (A; CYP1A), 3-OHmidazolam (B; CYP3A), 4-OH-diclofenac (C; CYP2C9), 4-OH-bupropion (D; CYP2B6), 1-OH-bufuralol (E; CYP2D6), and 4-OH-mephenytoin (F; CYP2C19), were measured by liquid chromatography/mass spectrometry. The CYP activity is presented as pmol metabolite per mg protein per minute (mean $\pm \mathrm{SD}$ ). Statistical significance was determined by applying the $t$-test or Welch's $t$-test. $Y$-axis is in the logarithmic scale. ns (nonsignificant), $* P<0.05, * * P<0.01$, and $* * * P<0.001$.

including measuring the activity of cytochrome P450 enzymes, measuring albumin secretion, and urea production in order to see if the observed correlation on the transcriptional level could be corroborated on the functional level.

The activity of cytochrome P450 enzymes was measured by incubating hPSC-HEP day 29 and hphep with a cocktail of substances, which are metabolized by CYP1A, CYP2D6, CYP2B6, CYP2C9, CYP3A, and CYP2C19 enzymes. The concentrations of the resulting metabolites were measured by liquid chromatography/mass spectrometry. Figure 6 shows the results for the different metabolites. For CYP1A, CYP3A, CYP2D6, and CYP2C19, the difference between their activities in hPSC-HEP compared to hphep is not significant. However, for CYP2C9 and CYP2B6, hphep showed significantly higher activities than hPSC-HEP $(P<0.05$ for CYP2C 9 and $P<0.01$ for CYP2B6). In order to confirm the protein expression of CYP3A4, immunocytochemical stainings for CYP3A4 were performed in hPSC-HEP (Figure 7) and indicated substantial CYP3A4 expression in a subpopulation of hPSC-HEP, which is reminiscent of metabolic zonation found in the liver lobe.

Results from the albumin secretion assay revealed comparable levels of albumin secretion between hPSC-HEP and hphep (Figure 8A), indicating a mature hepatocyte feature. The difference between hPSC-HEP and hphep was not significant because of substantial interindividual variation between the hphep donors, resulting in a high standard deviation in comparison to the difference in the mean values between hPSC-HEP and hphep. On the other hand, urea secretion was significantly lower in hPSC-HEP compared to hphep (Figure $8 \mathrm{~B}$ ) indicating that this function requires further improvement.

\section{DISCUSSION}

Human PSC-HEP have the potential to compensate for the shortage and drawbacks of currently used hepatocyte models because these cells share many features and functions with their in vivo counterparts, and their supply is virtually unlimited. They have the potential to serve as models for diseases such as nonalcoholic fatty liver disease/nonalcoholic steatohepatitis, as viral infection models, for drug screening,

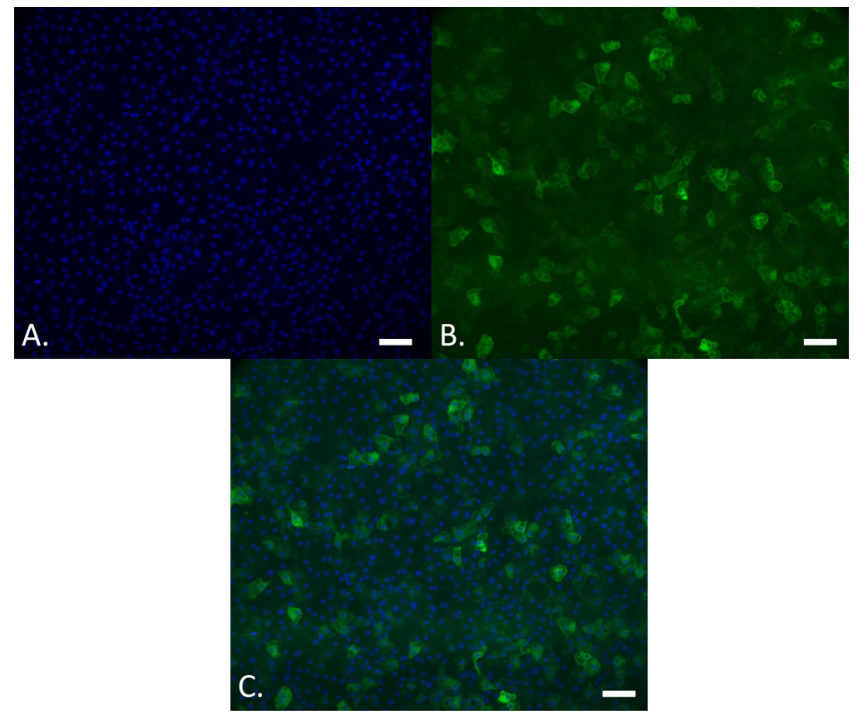

Figure 7. Representative micrographs illustrating the expression of CYP3A4 in hPSC-HEP (derived from cell line ChiPSC6b) on day 29 $(20 \times$ magnification, scale $50 \mu \mathrm{m}$ ). (A) 4',6-Diamidino-2-phenylindole (DAPI) staining the nuclei, (B) staining for CYP3A4, and (C) merge of DAPI and CYP3A4.

and in regenerative medicine. Although hPSC-HEP may be already good-to-go for some areas, some functions still need to be improved, for example, to allow the hPSC-HEP to be useful for drug metabolism and hepatotoxicity studies as discussed below.

In the present study, we investigated hPSC-HEP derived with an advanced differentiation protocol that allows generating homogenous hepatocyte cultures from a large panel of hPSC lines without the need to adapt the protocol to individual lines. $^{7-9}$ The differentiation protocol appears to impose tight control of the differentiation process, as demonstrated by the highly synchronized expression of lineage-specific genes when deriving hepatocytes from multiple hPSC lines. ${ }^{8}$

In order to evaluate the maturation status of the hPSC-HEP and also in order to identify novel targets to improve the 

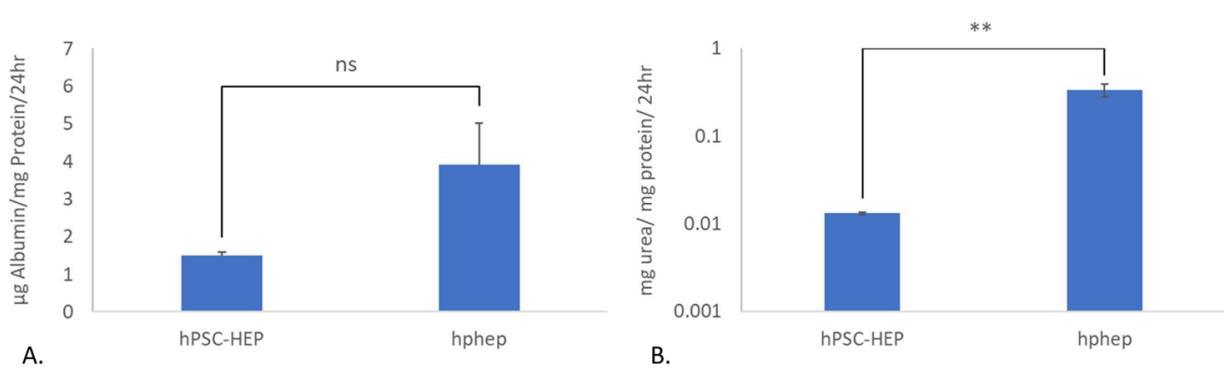

Figure 8. Analysis of hPSC-HEP day $29(N=2$ cell lines) and hphep $24 \mathrm{~h}$ post-thaw/plating $(N=4$ donors). Functions measured after $24 \mathrm{~h}$ of incubation: (A) albumin secretion presented as $\mu \mathrm{g}$ albumin $/ \mathrm{mg}$ protein $/ 24 \mathrm{~h}$ (mean $\pm \mathrm{SD}$ ) and (B) urea production presented as $\mathrm{mg}$ urea/mg protein $/ 24 \mathrm{~h}$ (mean $\pm \mathrm{SD}, Y$-axis in the logarithmic scale).

functionality of hPSC-HEP, we performed extensive transcriptomics analysis on our dataset (E-MTAB-5367), which covered the complete hepatocyte differentiation procedure and included AL reference samples. Because our dataset did include AL samples, but not FL samples, we merged it with a dataset retrieved from the GEO database, which included both $\mathrm{AL}$ and FL. As AL samples overlapped across the datasets, these were used to successfully guide the batch effect reduction in the merged dataset. We applied both correlation and similarity analyses between pairwise combinations of all time points during hepatocyte differentiation to the FL and $\mathrm{AL}$ samples. Notably, our results showed that the hPSC-HEP used in this study have a significantly higher correlation with AL than with FL ( 0.83 and 0.70 , respectively), indicating an adult rather than a fetal transcriptional phenotype of the hPSC-HEP, which is in contrast to previous reports using other hepatocyte differentiation protocols. ${ }^{2,4}$ In line with the Spearman's correlation results, the gene expression in hPSC-HEP was found to be more similar to AL than to FL (75 and 56\%, respectively).

Moreover, we analyzed several functions in order to further investigate the maturity status of the hPSC-HEP including CYP activities, albumin secretion, and urea production. Notably, differences of CYP1A, CYP3A, CYP2C19, and CYP2D6 enzyme activities between hPSC-HEP and hphep were nonsignificant, which may in part result from the high interindividual variation in CYP activities in hphep displayed by high standard deviations (Figure 6). Because the activity assay does not allow the discrimination between the different isoenzymes CYP3A4, 3A5, and 3A7, one needs additional tests to determine the expression levels of the different isoenzymes. Noteworthy, CYP3A4 is not differentially expressed between hPSC-HEP and hphep on the mRNA level (therefore, CYP3A4 is not listed in Table S3), which indicates an adult feature of hPSC-HEP because the CYP3A4 expression is not present in the FL. ${ }^{12}$ CYP3A4 protein expression in hPSC-HEP was also confirmed by immunostainings (Figure 7), and as reported previously, the CYP3A4 protein expression is highly correlated with its enzyme activity. ${ }^{13}$ In addition, the mRNA expression of the fetal isoenzyme CYP3A7 was lower in hPSCHEP than in AL (Table S3), which supports the finding that hPSC-HEP appear to have more adult-like features.

Another important hepatocyte feature, albumin secretion, was also detected in hPSC-HEP at levels comparable to hphep, supporting the notion of a partially adult phenotype of the hPSC-HEP (Figure 8A). In contrast, urea secretion in hPSCHEP still needs to be improved (Figure 8B). Another interesting finding was that the day 14 samples in the differentiation process (referred to as hepatoblasts) showed higher correlation with AL than with FL (0.78 and 0.70, respectively). A potential explanation could be that the FL harbors other cell types besides hepatoblasts, such as hematopoietic cells, which are not present in the in vitroderived hepatoblast population. ${ }^{14}$

Hitherto, hPSC-HEP have generally been classified as fetal or immature hepatocytes based on their restricted functionality and their expression of fetal genes. However, the correlation and similarity results presented in this study suggest that the gap between AL and hPSC-HEP has been greatly reduced regarding hPSC-HEP derived with the differentiation protocol used in this study. An extracellular matrix overlay and the advanced medium composition utilized in the differentiation protocol contribute to achieve more mature features in the hPSC-HEP compared to previous protocols. ${ }^{7}$ The matrix overlay, which is placed on top of the cells during the differentiation procedure, resembles the sandwich-culture configuration in which hepatocytes are cultured between two layers of extracellular matrix components such as collagen. Also, other reports confirm that the use of extracellular matrix components or the application of defined factors in the differentiation process may help in improving the functionality of stem cell-derived hepatocytes. 3,15

Another important finding in the present study was that only 11 genes were differentially expressed between hPSC-HEP day 25 and day 30, indicating a stable phenotype of the hPSC-HEP toward the later stages of differentiation. This may be attributed to the matrix overlay because this culturing technique previously has been shown to contribute to both longevity and retention of in vivo hepatocyte features in cultured primary hepatocytes. ${ }^{16}$

In our previous study, we found that hPSC-HEP derived from all six hPSC lines displayed a substantial expression of genes involved in xenobiotic metabolism, expressed serum proteins such as albumin and $\alpha 1$-antitrypsin, and were capable of glycogen storage. ${ }^{8}$ In the present study, we found that the pathways involved in the essential hepatocyte functionality, ${ }^{17}$ such as "complement and coagulation cascades," "drug induction of bile acid pathway," and "oxidation by cytochrome P450," in addition to biological process terms involved in the functionality of hepatocytes, ${ }^{17}$ such as "steroid metabolism" and "bile acid secretion," were enriched for hPSC-HEP. However, we also observed that $22 \%$ of the total $\mathrm{ADME}$ genes were downregulated in hPSC-HEP compared to AL. In accordance with this, we found CYP2D6, CYP2B6, and CYP2C19 enzyme activities to be lower in hPSC-HEP compared to hphep (Figure 6). All together, these results suggest a high similarity between the in vivo and in vitro 
hepatocytes but also reveal functions that require further improvement in hPSC-HEP.

Notably, the hPSC-HEP that have been investigated in this study mirror many functions of bona fide hepatocytes. ${ }^{17}$ Despite this significant progress compared to earlier reports, ${ }^{7}$ some functions still need to be improved. The major differences between hPSC-HEP and AL regarding ADME genes were the expression of the genes SLCO1B1, SLCO1B3, CYP2C8, CYP2E1, CYP2C9, CYP2A6, and CYP2B6 from the core ADME list, in addition to $A D H 1 B, C E S 1, A O X 1, A D H 1 A$, $A D H 1 C$, and PON1 from the extended ADME list. ${ }^{17}$ These genes showed lower expression in hPSC-HEP compared to AL. In our previous study, the genes CYP2E1, CYP2C9, CYP2A6, CYP2B6, and AOX1 were reported to belong to the same module (i.e., highly connected in a protein interaction network). ${ }^{9}$ Pathway analysis revealed the association of some of these genes with the "constitutive androstane receptor pathway" where the constitutive androstane receptor (CAR, NR113) is involved in regulating the transcription of CYP2C9, CYP2A6, and CYP2B6. Interestingly, both CAR and the coregulator peroxisome proliferator-activated receptor gamma, coactivator 1 alpha 2 (PPARGC1A), are downregulated in hPSC-HEP compared to AL, which might cause the low expression of these downstream genes. ${ }^{18}$

Only four genes (STC1, LINC00261, ACE2, and COL5A2) were differentially expressed among all investigated groups, of which COL5A2, STC1, and ACE2 were downregulated in AL compared to hPSC-HEP. These genes have previously been reported to be involved in inter alia, regulation of cell differentiation, proliferation, adhesion, liver injury, and tumorigenesis. ${ }^{19-21}$ The TF enrichment analysis identified seven TFs (AR, BACH1, FOXA1, FOXA2, FOXM1, JUN, and SOX2) that may regulate these genes, of which only the $A R$ was differentially expressed between hPSC-HEP and AL, which may account for the observed difference. This result indicates that AR may be an interesting candidate to target in for further protocol optimization.

Importantly, four TFs (CDX2, CLOCK, HIF1A, and NR1H3) were identified with enriched binding sites for genes whose expression differed significantly only between hPSC-HEP and AL. These TFs, in addition to the affected pathways that were enriched for the differentially expressed genes between hPSC-HEP and AL, need to be further explored to identify approaches to adjust the gene expression in hPSC$\mathrm{HEP}$ in order to reach the functionality levels of their in vivo counterparts. One suitable strategy may be the use of inhibitors or activators to downregulate or upregulate, respectively, the expression of deviated genes.

Remarkably, most of the genes that were differentially expressed only in the comparisons, hepatoblasts versus hPSCHEP and hPSC-HEP versus AL, were upregulated in hPSCHEP compared to both hepatoblasts and AL. Notably, the TFs regulating these genes, $C D X 2$ and $K L F 5$, were also upregulated in hPSC-HEP compared to both hepatoblasts and AL. These colon-associated TFs were also identified in hepatocyte-like cells in previous reports, implying that the cell population expresses features from different cell types ${ }^{2}$ and are targets in ongoing studies.

Interestingly, a potential novel biomarker was also identified for DE. The noncoding transcript RP4-559A3.6 with an unknown function shows a high FC level during the transition from hPSCs to DE. RP4-559A3.6 partially overlaps the leftright determination factor 2 (LEFTY2) sequence. LEFTY2 is a nodal antagonist activated by nodal to regulate the differentiation process toward the different germ layers. ${ }^{22}$

\section{CONCLUSIONS}

Taken together, our results demonstrated higher correlation and higher similarity in gene expression levels between hPSCHEP and AL than between hPSC-HEP and FL. Moreover, functional data confirms important mature hepatocyte features of hPSC-HEP. Our results also showed that a fraction of the ADME genes differed significantly between hPSC-HEP and $\mathrm{AL}$, indicating that xenobiotic metabolism requires further improvement in hPSC-HEP. In addition, we identified abnormal gene expression and deviations in hepatocyte pathways and TFs that may regulate these deviations. Thus, on the transcriptome level, several putative targets with great potential for improvement of future hepatocyte differentiation protocols were identified. Both chemical and genetic manipulations of these factors are promising approaches to further improve the functionality of hPSC-HEP. A DNA methylome study is ongoing to gain new insights into the complexity of the hPSC-HEP, which likely will help in correcting some of the remaining deviating functions of hPSC-HEP and make their phenotype even more similar to human adult hepatocytes.

\section{MATERIALS AND METHODS}

Microarray Datasets. Two different transcriptomics datasets were used in this study. The differentiation analyses are based on a transcriptomics dataset from hepatocyte differentiation of hPSCs generated in a previous study. ${ }^{9}$ This dataset includes global gene expression profiles from six male (XY) hPSC lines, which were differentiated into hepatocytes by applying a prototype of Cellartis Hepatocyte Differentiation Kit provided by Takara Bio Europe AB (www.clontech.com) and two AL samples, as described previously. ${ }^{8}$ In short, samples from five defined differentiation stages (hPSC, DE, hepatoblasts, hPSC-HEP day 25, and hPSC-HEP day 30) were analyzed using the GeneChip Human Transcriptome Array 2.0 (Affymetrix). Duplicate samples were generated in two separate differentiation experiments from six different stem cell lines [three human embryonic stem cell (hESC) lines and three human induced pluripotent stem cell (hiPSC) lines; $N=$ 2 batches per cell line]. In addition, two AL tissue samples ( $N$ $=2$ donors) from $\mathrm{XY}$ donors were included as reference samples. To compare the hPSC-HEP to tissue samples from the FL, an additional dataset containing $14 \mathrm{FL}$ tissue samples and $92 \mathrm{AL}$ tissue samples was used. ${ }^{23}$ Both datasets are publicly available through the ArrayExpress database, accession number E-MTAB-5367, and the GEO database, accession number GSE61279.

Overview of the Bioinformatics Analyses. Extensive bioinformatics analyses were performed to investigate the gene expression patterns at defined developmental stages during the differentiation of hPSCs to the hepatocyte lineage. These analyses were composed following our developed transcriptomics data analysis framework proposed in Ulfenborg et al. ${ }^{24}$ (Figure 9).

Merging of Two Different Microarray Datasets. To benchmark the hPSC-HEP and define their level of maturation, they were compared to samples from FL and $\mathrm{AL}$ tissues. To this end, publicly available transcriptomics data from the GEO database (GSE61279) containing samples from 


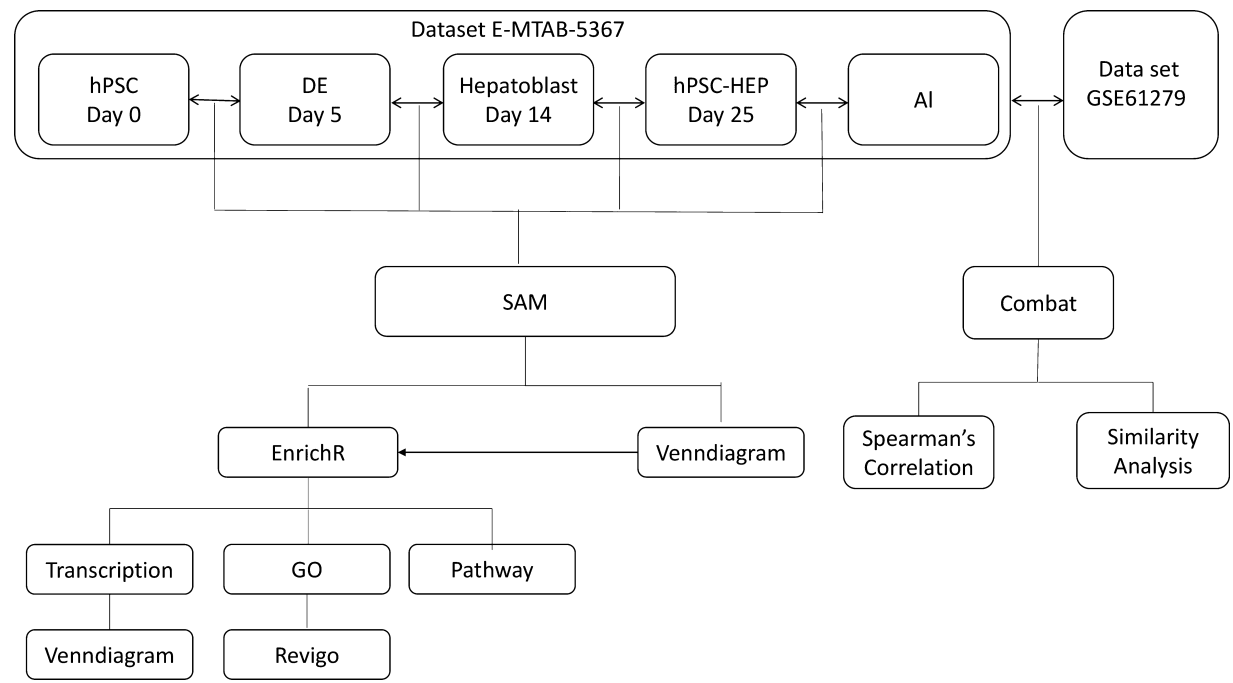

Figure 9. Schematic overview of the bioinformatic analysis performed on the datasets E-MTAB-5367 (ArrayExpress database) and GSE61279 (GEO database).

both AL and FL were used. Only human FL samples from XY donors and human AL samples from XY aged 20-38 years with "accident" as the cause of death were selected for this analysis to minimize variability and collect a homogenous set of control samples. In total, six FL tissue samples and seven AL samples were selected from this dataset. The samples from dataset E-MTAB-5367 (excluding hPSC-HEP day 30, constituting one data batch) were merged with the selected samples from dataset GSE61279 (constituting the second data batch). To reduce batch differences, batch effect correction was performed on the merged dataset, applying the ComBat() function from the sva package in $\mathrm{R}^{10,25}$ There are AL samples in both datasets, and these were used as overlapping samples to guide the batch reduction procedure. To evaluate the normalization and reduction of batch effects in the merged dataset, the distribution of expression values across the samples before and after the batch effect correction was investigated using boxplots. A hierarchical clustering analysis was also performed on the merged dataset, applying the hclust() method from the genefilter package in $\mathrm{R}$. The merged dataset with normalized values is available in Supporting Information, Table S8.

Spearman's Correlation Analysis. To assess the level of maturation of the in vitro differentiated hepatocytes, the correlation between the global transcriptional profiles of the hPSC-derived hepatocytes and the FL and AL samples was analyzed by applying cor.test() with a confidence interval of 95\% in R to calculate the Spearman's correlation on the mean values of the following groups: hPSC, DE, hepatoblasts and hPSC-HEP day 25 with the mean value of each of the following groups, FL week 8-9, FL week 11-12, FL week 21, and AL.

Similarity in Gene Expression between Developmental Stages in Vitro and in Vivo. Similarity between transcriptional profiles of the developmental stages hPSC, DE, hepatoblasts, and hPSC-HEP day 25 and FL and AL samples (FL week 8-9, FL week 11-12, FL week 21, and AL) was assessed as described in the study by Synnergren et al. ${ }^{26}$ where genes with an FC $<1.5$ were considered as similarly expressed. To exclude transcripts with high variability, only genes with coefficient of variance $(\mathrm{CV})<30 \%$ between the biological replicates were included in this analysis. The number of transcripts that passed the above criteria was counted as similarly expressed genes.

Identification of Differentially Expressed Genes. To identify genes, which show differential expression during the transitions between the studied hepatocyte developmental stages, SAM data in the Siggenes package in R was applied on dataset E-MTAB-5367. Pairs of samples from the in vitro hepatocyte differentiation representing defined developmental stages were analyzed to identify differentially expressed genes during each transition as well as between hPSC-HEP and the reference samples (AL) (Figure 9).

Genes with an FDR $<0.05$ and FC $>3$ were considered as significantly differentially expressed. In addition, differentially expressed genes with an exceptionally high FC $(\geq 20)$ were also identified. Moreover, ADME genes, including a set of core $\mathrm{ADME}$ genes and an extended list of $\mathrm{ADME}$ genes, as categorized by PharmaADME (http://www.pharmaadme.org), were compared to the list of differentially expressed genes between hepatoblasts versus hPSC-HEP day 25 and hPSCHEP day 25 versus AL and explored in more detail.

To investigate the overlap between the differentially expressed genes from each transition, the Venndiagram package in $\mathrm{R}$ was applied using the lists of differentially expressed genes from the following comparisons: hPSC versus DE, DE versus hepatoblasts, hepatoblasts versus hPSC-HEP day 25, and hPSC-HEP day 25 versus AL. Only 11 genes, with no obvious connection to hepatocyte functionality, were differentially expressed between hPSC-HEP day 25 and hPSC-HEP day 30, and therefore, the samples from hPSC-HEP day 30 were excluded from further analyses.

GO Enrichment Analysis of the Differentially Expressed Genes. To investigate the functional properties of the genes that show differential expression in each transition, a GO enrichment analysis was performed by applying the EnrichR software on dataset E-MTAB-5367. ${ }^{11}$ Biological processes with adjusted $P$-value $<0.05$ were considered. The results from the GO enrichment analysis were visualized by applying the Revigo software. ${ }^{27}$

Pathway Enrichment Analysis. The differentially expressed genes identified from each transition in dataset EMTAB-5367 were applied to the EnrichR software to detect significantly enriched pathways. ${ }^{11}$ Pathways with adjusted $P$ - 
value $<0.05$ from the Wikipathways database were considered in this analysis.

TF Enrichment Analysis. To explore putative mechanisms regulating the differentiation toward the hepatocyte lineage, a TF enrichment analysis was performed on dataset E-MTAB5367 using the identified sets of differentially expressed genes from each transition, hPSC-DE cells, DE-hepatoblasts, and hepatoblasts-hPSC-HEP day 25, and from the comparison hPSC-HEP day 25-AL by applying the EnrichR software. ${ }^{11}$ EnrichR scans the versatile TF databases to determine if the submitted gene list includes genes with binding sites for known TF. The software returns the TF whose target sites are enriched in the group of input genes, including a significance measure. Results from the TF interaction database ChIP Enrichment Analysis (ChEA_2016) with adjusted P-value < 0.05 were considered. A Venn diagram analysis was applied on the set of TF identified with enriched binding sites for the differentially expressed genes to reveal overlap between different transitions. The identified TFs in each transition were compared to the lists of the differentially expressed genes to determine if they are differentially expressed in our dataset and thus, a putative regulator of the transition.

Immunocytochemistry. hPSC-HEP were stained on day 29 for CYP3A4 as described previously. ${ }^{8}$ The antibodies, antihuman CYP3A4 Rabbit-IgG (cat. no. PAP011, Cypex) and Donkey anti-rabbit-IgG-Alexa 488 (cat. no. A-21206, Thermo Fisher Scientific), were diluted 1:200 and 1:1000, respectively.

Activity Assay for CYP Enzymes. The activities of the enzymes CYP1A, CYP3A, CYP2C9, CYP2C19, and CYP2D6 were measured in hPSC-HEP derived from the six hPSC-lines (on day 29 after start of differentiation) and in cryoplateable human primary hepatocytes from four different donors (BioIVT, Frankfurt am Main, Germany), as described previously. ${ }^{7,8}$ Briefly, the cells were incubated for $2 \mathrm{~h}$ with a cocktail of CYP substrates, $10 \mu \mathrm{M}$ phenacetin, $10 \mu \mathrm{M}$ bufuralol, $10 \mu \mathrm{M}$ diclofenac, $10 \mu \mathrm{M}$ bupropion, $50 \mu \mathrm{M}$ mephenytoin, and $5 \mu \mathrm{M}$ midazolam, metabolized by CYP1A, CYP2D6, CYP2C9, CYP2B6, CYP2C19, and CYP3A4, respectively. The human primary hepatocytes were plated and cultured for $20 \mathrm{~h}$ in total post-thaw, including the CYP activity assay. The concentrations of the produced metabolites (paracetamol, 1-OH-bufuralol, 4-OH-diclofenac, 4-OH-bupropion, 4-OH-mephenytoin, and 3-OH-midazolam) were measured by liquid chromatography/mass spectrometry performed at Pharmacelsus GmbH (Saarbrücken, Germany).

Detection of Albumin Secretion. Albumin secretion was measured in duplicate using the Albuwell ELISA kit (Cat. no. 1004, Exocell) according to the instruction of the manufacturer. Samples were collected from hPSC-HEP derived from ChiPSC6b and ChiPSC18 (on day 29 after start of differentiation) after $24 \mathrm{~h}$ of incubation in their standard maintenance medium Cellartis hepatocyte maintenance medium (cat. no. Y30051; Takara Bio Europe AB). Cryoplateable human primary hepatocytes from three different donors were plated and incubated for $24 \mathrm{~h}$ in InvitroGro CP medium (BioIVT). Twenty fours post-thaw, the medium was changed to Cellartis power primary hepatocyte medium (cat. no. Y20020, Takara Bio Europe AB), and samples were collected $24 \mathrm{~h}$ later. Results were calculated from the log-log regression obtained by the fit of the standard curve and normalized to protein content and are presented in $\mu \mathrm{g}$ albumin/mg protein.
Urea Determination by Colorimetric Quantification. The determination of urea was assayed using the QuantiChrom UREA Assay Kit (cat. no. DIUR-500, Bioassay Systems) according to the recommendation of the manufacturer with minor modifications.

Samples were collected from hPSC-HEP derived from ChiPSC6b and ChiPSC18 (on day 29 after start of differentiation) after $24 \mathrm{~h}$ of incubation in their standard maintenance medium Cellartis hepatocyte maintenance medium (cat. no. Y30051; Takara Bio Europe AB) supplemented with $5 \mathrm{mM}$ ammonium chloride and with a control group incubated in a medium without ammonium chloride. Cryoplateable human primary hepatocytes from three different donors were plated and incubated for $24 \mathrm{~h}$ in InvitroGro CP medium (BioIVT). Twenty four hours postthaw, the medium was changed to Cellartis power primary hepatocyte medium (cat. no. Y20020, Takara Bio Europe AB) supplemented with $5 \mathrm{mM}$ ammonium chloride (a control group was incubated in a medium without ammonium chloride), and samples were collected 24 h later. A standard curve was constructed with the following concentrations of urea: 5, 2.5, 1.25, 0.625, 0.312, 0.156, and $0.078 \mathrm{mg}$. Equal volumes of reagent A and reagent B were prepared, and $200 \mu \mathrm{L}$ was added to each sample. The samples were incubated for 50 min at room temperature. After incubation, the absorbance of all samples was read at $430 \mathrm{~nm}$. Raw data was blank-corrected using the absorbance of the control samples. The results were calculated from the linear regression obtained by the fit of the standard curve and normalized to protein content and are presented in $\mathrm{mg}$ urea/mg protein.

Statistical Analysis. To determine if there is a significant statistical difference between the compared groups, we applied $t$-test for groups with equal variances or groups that had different variances, but the group with the largest variance included fewer samples. ${ }^{28}$ Otherwise we applied the Welch's $t$ test to ensure the highest statistical power. As demonstrated previously, $t$-test is feasible with an extremely small sample size $(N \leq 5)$. $^{28}$

\section{ASSOCIATED CONTENT}

\section{Supporting Information}

The Supporting Information is available free of charge at https://pubs.acs.org/doi/10.1021/acsomega.9b03514.

Boxplot showing the distribution of the expression values in the merged and normalized datasets E-MTAB5367 (red) and GSE61279 (blue); expression profiles of region A2 genes; and expression profile of region A11 genes (PDF)

Lists of differentially expressed genes $(\mathrm{FC}>3)$ between the different time points during the hepatic differentiation and the controls; differentially expressed genes with $\mathrm{FC}$ values $>20$ between sequential developmental stages; $\mathrm{ADME}$ genes differentially expressed between hPSC-hESC day 25 and AL; ADME genes which are differentially expressed between hepatoblast (day 14) and hPSC-hESC day 25; gene lists from the different regions in the Venn diagram; GO enrichment analysis for biological process terms for the differentially expressed genes between the transitions: hPSC-DE (d0vsd5), DE-hepatoblasts (d5vsd14), hepatoblastshPSC-HEP day 25 (d14vsd25), and hPSC-HEP day 25-AL (d25vsAL); pathways enriched for differentially 
expressed genes between sequential development stages during hepatic differentiation; and normalized and batch-corrected data from the merged datasets EMTAB-5367 (ArrayExpress database) and GSE61279 (GEO database) (XLSX)

\section{AUTHOR INFORMATION}

\section{Corresponding Author}

Nidal Ghosheh - School of Bioscience, Systems Biology Research Center, University of Skövde, 54128 Skövde, Sweden; (1) orcid.org/0000-0003-2942-6702; Phone: +46 (0) 76215 56 54; Email: nidal.ghosheh@his.se; Fax: +46 (0) 3175809 10

\section{Authors}

Barbara Küppers-Munther - Takara Bio Europe AB, 41346 Gothenburg, Sweden

Annika Asplund - Takara Bio Europe AB, 41346 Gothenburg, Sweden

Christian X. Andersson - Takara Bio Europe AB, 41346 Gothenburg, Sweden

Petter Björquist - VeriGraft AB, 41346 Gothenburg, Sweden

Tommy B. Andersson - Cardiovascular Renal and Metabolism, Innovative Medicines and Early Development Biotech Unit, AstraZeneca, Mölndal 431 83, Sweden; Department of Physiology and Pharmacology, Section of Pharmacogenetics, Karolinska Institutet, 17177 Stockholm, Sweden

Helena Carén - Sahlgrenska Cancer Center, Department of Pathology, Institute of Biomedicine, Sahlgrenska Academy, University of Gothenburg, 40530 Gothenburg, Sweden

Stina Simonsson - Institute of Biomedicine, Department of Clinical Chemistry and Transfusion Medicine, Sahlgrenska Academy, University of Gothenburg, 41345 Gothenburg, Sweden

Peter Sartipy - School of Bioscience, Systems Biology Research Center, University of Skövde, 54128 Skövde, Sweden; Late Stage Cardiovascular, Renal, and Metabolism, $R \& D$ BioPharmaceuticals, AstraZeneca, Mölndal 431 83, Sweden

Jane Synnergren - School of Bioscience, Systems Biology Research Center, University of Skövde, 54128 Skövde, Sweden

Complete contact information is available at:

https://pubs.acs.org/10.1021/acsomega.9b03514

\section{Author Contributions}

A.A., B.K.-M., C.X.A., H.C., T.B.A., J.S., N.G., P.B., P.S., and S.S. participated in the research design. N.G. conducted the experiments. A.A., B.K.-M., J.S., and N.G. performed the data analysis. A.A., B.K.-M., C.X.A., H.C., J.S., N.G., P.B., P.S., S.S., and T.B.A. wrote or contributed to the writing of the manuscript.

\section{Notes}

The authors declare the following competing financial interest(s): Authors Barbara Kppers-Munther, Annika Asplund , and Christian X. Andersson are employees of Takara Bio Europe AB. Authors Tommy B. Andersson and Peter Sartipy are employees of AstraZeneca.

\section{ACKNOWLEDGMENTS}

This work was supported by Takara Bio Europe AB (Gothenburg, Sweden), AstraZeneca (Mölndal, Sweden), and the University of Skövde, Sweden, under grants from the
Knowledge Foundation [2012/0310], [2013/89], and [2014/ 0301].

\section{ABBREVIATIONS}

ABC, ATP-binding cassette; ACE2, angiotensin I converting enzyme 2; ADME, absorption, distribution, metabolism, and excretion; AFP, alpha fetoprotein; AL, adult liver; AOX1, aldehyde oxidase 1; APELA, apelin receptor early endogenous ligand; $\mathrm{AR}$, androgen receptor; $\mathrm{BACH} 1, \mathrm{CNC}$ homolog 1; CAR, constitutive androstane receptor; $\mathrm{CDH} 17$, cadherin 17; CDX2, caudal Type homeobox 2; CES1, carboxylesterase 1; CLOCK, clock circadian regulator; COL5A2, collagen type V alpha 2 chain; CV, coefficient of variance; CYP, cytochrome P450; DAPI, 4',6-diamidino-2-phenylindole; DE, definitive endoderm; DOCK11, dedicator of cytokinesis 11; DUSP6, dual specificity phosphatase 6; FC, fold change; FDR, false discovery rate; FL, fetal liver; FOX, Forkhead box; FST, follistatin; GEO, Gene Expression Omnibus; GO, gene ontology; GSTA, glutathione $S$-transferase alpha; HIF1A, hypoxia inducible factor 1 subunit alpha; HNF, hepatocyte nuclear factors; hphep, human primary hepatocyte; hPSC, human pluripotent stem cells; hPSC-HEP, human pluripotent stem cell derived hepatocytes; JUN, jun oncogene; KLF5, Kruppel like factor 5; LCT, lactase; LEFTY2, left-right determination factor 2; LINC, long intergenic non-protein coding RNA; MUC13, mucin 13; NANOG, Nanog homeobox; NR1H3, liver X receptor alpha; PON1, paraoxonase 1; PPARGC1A, peroxisome proliferator-activated receptor gamma, coactivator 1 alpha 2; PROX1, prospero homeobox 1; RHGAP28, Rho GTPase activating protein 28; SAM, significance analysis of microarray; $\mathrm{RT}$, room temperature; SAMHD1, SAM domain and HD domain 1; SLCO, solute carrier organic anion transporter family; SOX2, sex determining region Y-box 2; STC1, stanniocalcin 1; STOM, stomatin; $\mathrm{TF}$, transcription factor; TXNIP, thioredoxin interacting protein; XY, male

\section{REFERENCES}

(1) Zhao, D.; Chen, S.; Duo, S.; Xiang, C.; Jia, J.; Guo, M.; et al. Promotion of the efficient metabolic maturation of human pluripotent stem cell-derived hepatocytes by correcting specification defects. Cell Res. 2013, 23, 157-161.

(2) Godoy, P.; Schmidt-Heck, W.; Natarajan, K.; Lucendo-Villarin, B.; Szkolnicka, D.; Asplund, A.; et al. Gene networks and transcription factor motifs defining the differentiation of stem cells into hepatocytelike cells. J. Hepatol. 2015, 63, 934-942.

(3) Szkolnicka, D.; Hay, D. C. Concise Review: Advances in Generating Hepatocytes from Pluripotent Stem Cells for Translational Medicine. Stem Cells 2016, 34, 1421-1426.

(4) Baxter, M.; Withey, S.; Harrison, S.; Segeritz, C.-P.; Zhang, F.; Atkinson-Dell, R.; et al. Phenotypic and functional analyses show stem cell-derived hepatocyte-like cells better mimic fetal rather than adult hepatocytes. J. Hepatol. 2015, 62, 581-589.

(5) Zhou, Z.; Xu, M.-J.; Gao, B. Hepatocytes: a key cell type for innate immunity. Cell. Mol. Immunol. 2016, 13, 301-315.

(6) Si-Tayeb, K.; Lemaigre, F. P.; Duncan, S. A. Organogenesis and Development of the Liver. Dev. Cell 2010, 18, 175-189.

(7) Asplund, A.; Pradip, A.; van Giezen, M.; Aspegren, A.; Choukair, H.; Rehnström, M.; et al. One Standardized Differentiation Procedure Robustly Generates Homogenous Hepatocyte Cultures Displaying Metabolic Diversity from a Large Panel of Human Pluripotent Stem Cells. Stem cell reviews 2016, 12, 90-104.

(8) Ghosheh, N.; Olsson, B.; Edsbagge, J.; Kuppers-Munther, B.; Van Giezen, M.; Asplund, A.; et al. Highly Synchronized Expression of Lineage-Specific Genes during In Vitro Hepatic Differentiation of 
Human Pluripotent Stem Cell Lines. Stem Cells Int. 2016, 2016, 8648356.

(9) Ghosheh, N.; Kuppers-Munther, B.; Asplund, A.; Edsbagge, J.; Ulfenborg, B.; Andersson, T. B.; et al. Comparative transcriptomics of hepatic differentiation of human pluripotent stem cells and adult human liver tissue. Physiol. Genomics 2017, 49, 430.

(10) Turnbull, A. K.; Kitchen, R. R.; Larionov, A. A.; Renshaw, L.; Dixon, J. M.; Sims, A. H. Direct integration of intensity-level data from Affymetrix and Illumina microarrays improves statistical power for robust reanalysis. BMC Medical Genomics 2012, 5, 35.

(11) Kuleshov, M. V.; Jones, M. R.; Rouillard, A. D.; Fernandez, N. F.; Duan, Q.; Wang, Z.; et al. Enrichr: a comprehensive gene set enrichment analysis web server 2016 update. Nucleic Acids Res. 2016, 44, W90-W97.

(12) Hakkola, J.; Tanaka, E.; Pelkonen, O. Developmental expression of cytochrome P450 enzymes in human liver. Pharmacol. Toxicol. 1998, 82, 209-217.

(13) Watanabe, M.; Kumai, T.; Matsumoto, N.; Tanaka, M.; Suzuki, S.; Satoh, T.; et al. Expression of CYP3A4 mRNA is correlated with CYP3A4 protein level and metabolic activity in human liver. $J$. Pharmacol. Sci. 2004, 94, 459-462.

(14) Schmelzer, E.; Zhang, L.; Bruce, A.; Wauthier, E.; Ludlow, J.; Yao, H.-1.; et al. Human hepatic stem cells from fetal and postnatal donors. J. Exp. Med. 2007, 204, 1973-1987.

(15) Cameron, K.; Tan, R.; Schmidt-Heck, W.; Campos, G.; Lyall, M. J.; Wang, Y.; et al. Recombinant Laminins Drive the Differentiation and Self-Organization of hESC-Derived Hepatocytes. Stem Cell Rep. 2015, 5, 1250-1262.

(16) Swift, B.; Pfeifer, N. D.; Brouwer, K. L. R. Sandwich-cultured hepatocytes: anin vitromodel to evaluate hepatobiliary transporterbased drug interactions and hepatotoxicity. Drug Metabol. Rev. 2010, 42, 446-471.

(17) Hengstler, J. G.; Brulport, M.; Schormann, W.; Bauer, A.; Hermes, M.; Nussler, A. K.; et al. Generation of human hepatocytes by stem cell technology: definition of the hepatocyte. Exp. Opin. Drug Metabol. Toxicol. 2005, 1, 61-74.

(18) Timsit, Y. E.; Negishi, M. CAR and PXR: The xenobioticsensing receptors. Steroids 2007, 72, 231-246.

(19) Zhang, Z.; Chen, L.; Zhong, J.; Gao, P.; Oudit, G. Y. ACE2/ Ang-(1-7) signaling and vascular remodeling. Sci. China Life Sci. 2014, 57, 802-808.

(20) Yeung, B. H. Y.; Shek, F. H.; Lee, N. P.; Wong, C. K. C. Stanniocalcin-1 Reduces Tumor Size in Human Hepatocellular Carcinoma. PLoS One 2015, 10, No. e0139977.

(21) Verrecchia, F.; Mauviel, A.; Rossert, J. Blocking sp1 transcription factor broadly inhibits extracellular matrix gene expression in vitro and in vivo: implications for the treatment of tissue fibrosis. J. Invest. Dermatol. 2001, 116, 755-763.

(22) Meno, C.; Gritsman, K.; Ohishi, S.; Ohfuji, Y.; Heckscher, E.; Mochida, K.; et al. Mouse Lefty 2 and Zebrafish Antivin Are Feedback Inhibitors of Nodal Signaling during Vertebrate Gastrulation. Mol. Cell 1999, 4, 287-298.

(23) Bonder, M.; Kasela, S.; Kals, M.; Tamm, R.; Lokk, K.; Barragan, I.; et al. Genetic and epigenetic regulation of gene expression in fetal and adult human livers. BMC Genomics 2014, 15, 860.

(24) Ulfenborg, B.; Karlsson, A.; Riveiro, M.; Améen, C.; Åkesson, K.; Andersson, C. X.; et al. A data analysis framework for biomedical big data: Application on mesoderm differentiation of human pluripotent stem cells. PLoS One 2017, 12, No. e0179613.

(25) Walsh, C.; Hu, P.; Batt, J.; Santos, C. Microarray Meta-Analysis and Cross-Platform Normalization: Integrative Genomics for Robust Biomarker Discovery. Microarrays 2015, 4, 389-406.

(26) Synnergren, J.; Améen, C.; Jansson, A.; Sartipy, P. Global transcriptional profiling reveals similarities and differences between human stem cell-derived cardiomyocyte clusters and heart tissue. Physiol. Genom. 2012, 44, 245-258.

(27) Supek, F.; Bošnjak, M.; Škunca, N.; Šmuc, T. REVIGO summarizes and visualizes long lists of gene ontology terms. PLoS One 2011, 6, No. e21800.
(28) de Winter, J. C. F. Using the Student's t-Test with Extremely Small Sample Sizes. Practical Assessment, Research and Evaluation 2013, 18, 10 . 